\title{
Selectivity of Acrylic Acid Radiation Grafted Non-Woven Polypropylene Sheets towards Some Heavy Metals Ions
}

\author{
Dalia El-Sayed Hegazy \\ National Centre for Radiation Research and Technology, Cairo, Egypt \\ Email: Dalia_eahegazy@Yahoo.com
}

Received December 27, 2011; revised January 27, 2012; accepted February 5, 2012

\begin{abstract}
Graft polymerization has been considered as a general method for the modification of the physical and chemical properties of polymeric materials and of particular interest for synthesis of the hydrophilic membrane. In this study, hydrophilic carboxylic acid groups were introduced by radiation-induced grafting of acrylic acid (AAc) onto non-woven polypropylene fabric (NWPP). Different irradiation doses and (AAc) monomer concentrations were used to optimize grafting yield. Characterization and properties of the prepared graft copolymer were studied by employing X-ray Diffraction (XRD), Thermogravimetric Analysis (TGA), Scanning Electron Microscope (SEM ), Fourier Transform Infrared Spectrometry (FTIR) and differential scanning calorimeter (DSC). The prepared grafted materials were used for removing some heavy metals ions. The results showed that the non-woven PP grafted with (AAc) has good affinity toward $\mathrm{Cu}, \mathrm{Ni}$ and $\mathrm{Co}$.
\end{abstract}

Keywords: Radiation Grafting; Acrylic Acid; Characterization; Removal of Heavy Metals

\section{Introduction}

Water contamination with toxic metals causes serious human health problems. Enhanced metal separation techniques that require less energy with minimal impact on the environment are desirable. Many kinds of metal adsorbents including activated carbon, bentonite and synthetic polymers have been developed to remove toxic metals from streaming water [1]. Radiation-induced graft polymerization is widely used to produce high performance chemically active polymer materials for adsorption and separation processes on the basis of various commercial polymers available in different forms (films, fibers, resins, textiles, powders) [2,3].Various approaches for synthesizing polymer adsorbents have been developed, including direct graft polymerization of vinyl monomers already containing desirable functional groups. Adsorbents can be easily synthesized by radiation-induced graft polymerization. This technique creates an active radical in the trunk polymer by means of ionizing radiation and the polymeric graft chains propagate from the active radical in the trunk polymer [3,4]. Chemical initiation often brings about problems arising from local heating of the initiator, an effect that is absent in the formation of free-radical sites by radiation, which is only dependent upon the absorption of high-energy radiation. Due to large penetrating power of higher energy radiation, methods using radiation initiation provide the opportunity to carry out grafting at different depths of the base polymer matrix. Moreover, the molecular weight of the products can be better regulated in radiation techniques In general; synthesized polymers such as polyethylene and polypropylene have been used as trunk polymer due to their reactivity in the grafting process and high mechanical strength.

Non-woven materials have been increasingly used for a variety of applications in the world [5]. Non-woven materials are one of the products popularly used as reinforcements for many applications since they possess a good combination of strength, lightweight and flexibility compared to conventional materials [6]. Non-woven with specific surface properties are also of interest in many technical applications of the materials as the surface features affect adsorption, abrasion, adhesion, biocompatibility and other properties of the materials. The advantages of using nonwoven polypropylene NWPP as matrix are their relatively low processing temperature which is essential because of low thermal stability of natural fibers and their good properties and lower cost. For these increasing applications it is desirable to produce such nonwoven materials with well defined surface properties. Various techniques have been developed to modify the surface properties of nonwoven materials [7-9].

In recent years, the removal of hazardous heavy metals from water and soil environments and industrial waste streams has attracted considerable attention. Enhanced metal separation techniques that require less energy with 
minimal impact on the environment are desirable.

In the present work, radiation grafting of AAc monomer onto non-woven polypropylene fabrics is investigated to obtain homogeneous distributed graft copolymer chains with high grafting yield. Characterization and properties of the prepared graft copolymer are studied by (XRD), (TGA) and (DSC). Also, the study included measurements of the mechanical properties and determination of the tensile strength of these materials as a function of degree of grafting. The application of the prepared NWPP-AAc as adsorbent materials for waste water treatment from heavy and toxic metals is investigated.

\section{Experimental}

\subsection{Materials}

The materials used in this study were needle punched nonwovens made of melt blown nonwovens extruded from PP polymers. Film sheets were cut to the dimention of $4 \times 5 \mathrm{~cm}$. The monomer used throughout this work was acrylic acid (AAc) of laboratory grade chemicals of purity 99\% (Merck).

\section{2. $\gamma$-Irradiation}

Irradiation to the required doses was carried out using a ${ }^{60} \mathrm{Co} \gamma$-cell. The irradiation facility was constructed by the National Center for Radiation Research and Technology, Atomic Energy Authority of Egypt. Irradiation was carried out in air atmosphere at different irradiation doses.

\subsection{Radiation Grafting of Non-Woven Polypropylene}

NWPP sheets were washed with acetone, dried at $50^{\circ} \mathrm{C}$ in an electric oven until they attain a constant weight and then immersed in the monomer solution in glass tubes. Distilled water was used as a diluent of AAc. The graft copolymers were prepared by direct radiation grafting of AAc onto NWPP at different irradiation doses. The grafted films were removed and washed thoroughly with the proper solvent (hot distilled water) in order to extract the residual monomer and homopolymer which may be accumulated in the film and then soaked overnight in distilled water. These prepared grafted polymers were then dried in an electric oven at $40^{\circ} \mathrm{C}$ for $24 \mathrm{~h}$ and weighed. The degree of grafting was calculated using the following equation:

$$
\text { Degree of grafting }(\%)=\frac{W_{g}-W_{0}}{W_{0}} \times 100
$$

where $W_{0}$ and $W_{g}$ represent the weights of initial and grafted film, respectively.

To overcome homopolymerization of AAc, a small amount of transition metal ions are commonly used as inhibitors (1), ammonium ferrous sulfate (Mohr's salt) was added to the reaction medium $(0.5 \mathrm{wt} \%)$.

\subsection{Fourier-Transform Infrared (FTIR)}

To justify the introduction of functional groups onto the nonwoven PP, infrared spectroscopy analysis was carried out using a FTIR spectrometer. The infrared spectra of NWPP and grafted NWPP sheets were performed using Jasco 6300FT-IR.

\subsection{Thermogravimetric Analysis (TGA)}

TGA for the investigated samples were performed under nitrogen atmosphere at a flow rate $50 \mathrm{ml} / \mathrm{min}$. of pure nitrogen gas and the heating rate was $10^{\circ} \mathrm{C} / \mathrm{min}$. from ambient temperature up to $600^{\circ} \mathrm{C}$ using Perkin Elmer system of type pyris- 6 thermal analysis system.

\subsection{DSC Measurements}

Thermal parameters of the prepared grafted NWPP such as; melting temperature $\left(\mathrm{T}_{\mathrm{m}}\right)$ heat of melting and recrystallization $\left(\Delta H_{m}\right)$ were determined by differential scanning calorimeter (DSC) (Perkin Elmer equipped with a DSC-7 data station). Specimen ( $\approx 5 \mathrm{mg})$ of the sample is used for DSC measurements. Indium and Zinc standards were utilized to calibrate the temperature and thermal scale. The measurements were carried out in $\mathrm{N}_{2}$ atmosphere at a heating rate of $10^{\circ} \mathrm{C} \mathrm{min}^{-1}$.

\subsection{X-ray Diffraction Analysis (XRD)}

$\mathrm{X}$-ray diffraction (XRD) patterns were recorded using Shimadzu X-ray diffractometer (XRD-6000 model) equipped with X-ray tube at operating voltage of $40 \mathrm{kV}$ and an electric current of $30 \mathrm{~mA}$.

\subsection{Mechanical Properties}

Tensile tests were performed according to the ASTM D412-80 test method and were carried out using an Instron testing machine (Model H10KS from Hounsfield Co., England, loaded cell $10 \mathrm{KN}$ ) at $25^{\circ} \mathrm{C}$ and the crosshead speed was $10 \mathrm{~mm} / \mathrm{min}$. Five samples per formulation were tested.

\subsection{Metal Uptake Measurement}

The fixed weight of the prepared hydrogel was immersed in the metal feed solution of definite concentration (100 ppm). Merck atomic absorption standard solutions of these metals were used for the calibration process. The $\mathrm{pH}$ and temperature of metal feed solutions were adjusted before applying the hydrogels for treatment processes. The remaining metal ions in its feed solution were 
determined by an atomic absorption instrument (Unicam Model Solaar 929).

The metal uptake (E) was calculated as follows:

$$
\mathrm{E}(\mathrm{mmol} / \mathrm{g})=\frac{C_{i}-C_{f}}{W \times A \times 10}
$$

where $W$ is the weight of the dried grafted film, $A$ is the atomic weight of metal ion and $C_{i}$ and $C_{f}$ are the initial and remaining concentrations of metal ions in $\mathrm{mg} / \mathrm{l}(\mathrm{ppm})$, respectively. The total uncertainly for all experiments ranged from $3 \%-5 \%$.

\section{Results and Discussion}

The radiation grafting process of aqueous AAc is usually accompanied by undesirable homopolymerization. Metal salts such as ammonium ferrous sulphate (Mohr's salt), suppress homopolymer formation. $\mathrm{Fe}^{2+}$ is well known to have an inhibition effect on free radical graft polymerization. However, they can also cause a deactivation of the grafting process and lead to a decrease in grafting degree when taken in excess. In the present study, it was found that the addition of $0.5 \mathrm{wt} \%$ of Moher's salt to the aqueous AAc is enough to enhance radiation grafting and minimize the homopolymer formation.

\subsection{Effect of Monomer Concentration on the Grafting Yield}

Among the effective parameters for radiation grafting are the monomer concentration. The effect of AAc monomer concentration on the grafting yield was investigated as shown in Figure 1. It is clear that the degree of grafting increases by increasing AAc acid to reach a maximum at $30 \mathrm{wt} \%$ AAc. Thereafter, any increase in monomer concentration leads to decrease in the grafting degree. At 30 $\mathrm{wt} \% \mathrm{AAc}$, there is enough monomer available to reach into the reaction sites on the polymer free radicals, resulting in higher degrees of grafting. The excess of monomer concentration causes suppression of the monomer diffusion by increasing the viscosity of the grafting medium under the homopolymerization effect (the Tromsdorff effect) [10].

\subsection{Effect of Irradiation Dose}

Irradiation dose is also an important factor to optimize the grafting process and homogeneity of grafting distribution. This is due to the initiation of grafting sites and growing chain reactions are very dependent on the amount of free radicals formed. The effect of irradiation dose on the grafting yield of AAc onto NWPP was investigated and is shown in Figure 2. It is obvious that as the irradiation dose increases, the grafting degree increases. This trend can be explained to the fact that the increase in the

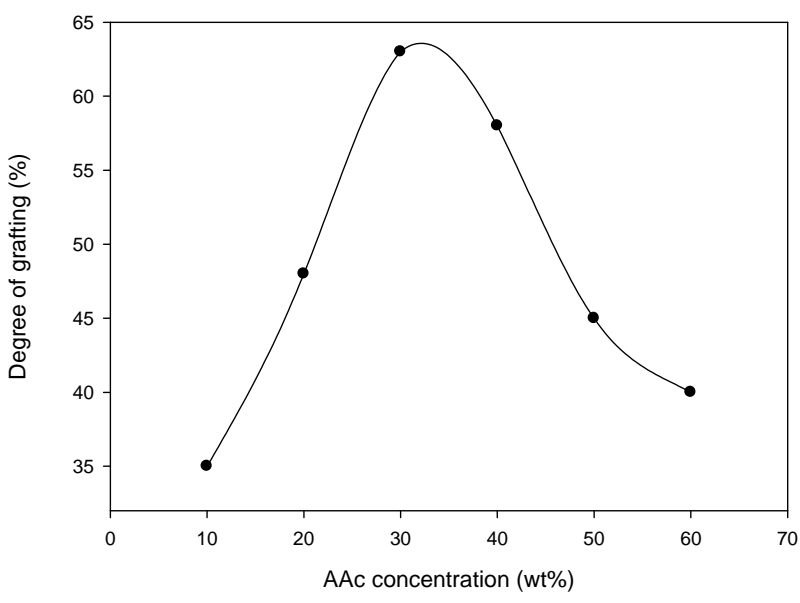

Figure 1. Effect of aqueous AAc concentration on the degree of grafting onto NWPP at irradiation dose (30 kGy).

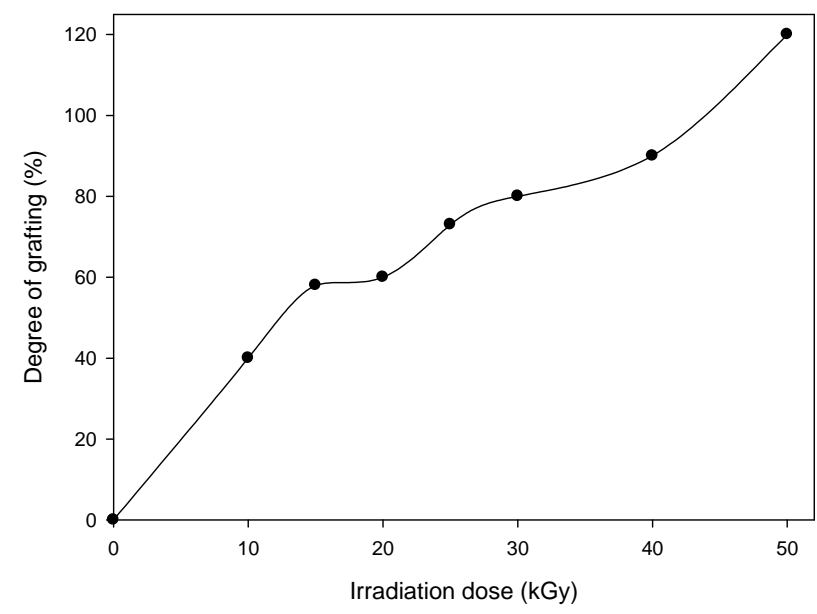

Figure 2. Effect of irradiation dose on the degree of grafting of AAc (30 wt \%).

irradiation dose leads to more free radical formation in the grafting sites which leads to an increase in the grafting yield. However, at irradiation doses above $30 \mathrm{kGy}$, the content of crosslinked insoluble homopolymer increased and it was difficult to extract it from the surface of NWPP sheets [11]. Also, a degraded rigid grafted NWPP sheets was formed at high irradiation dose.

\subsection{Swelling Behavior of the Grafted NWPP in Water}

From the practical point of view, the swelling properties and its ratio is very important property for its use in treatment process of waste water and diffusivity of metals through the grafted chains and functional groups. The swelling of the prepared NWPP-g-AAc copolymer in water was studied and the results are presented in Figure 3. It can be shown that as the degree of grafting increases, the swelling percent increases to reach a maximum at degree of grafting around $100 \%$. Thereafter, the swelling 


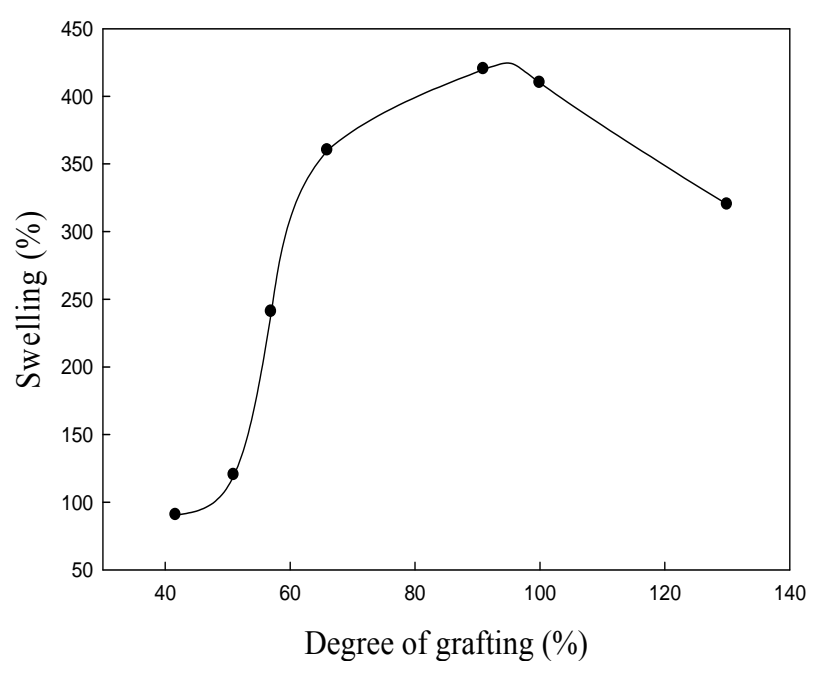

Figure 3. Swelling percent vs. degree of grafting percent for NWPP-g-AAc at swelling time $(24 \mathrm{~h})$.

percent tends to decrease with further increase in the grafting yield. As the irradiation dose increases, the increase in degree of grafting and cross-linking density which prevents a space for the AAc chain to swell freely in water and restricts and hindered the diffusivity of water through the cross-linked chains is expected. In other words, results showed clearly that the increase of irradiation dose during radiation grafting process enhanced the crosslinking via hydrogen bonding. As a consequence, the water uptake tends to decrease at high degrees of grafting.

\subsection{FTIR Analysis}

The FTIR spectra of the original NWPP and grafted NWPP films were studied and shown in Figure 4. FTIR spectrum of pure NWPP shows bands appearing at 28402800,1440 and $1380 \mathrm{~cm}^{-1}$, which are characteristic of the polypropylene structure. In case of NWPP-g-AAc, a new peak appears at around $1715.67 \mathrm{~cm}^{-1}$, which can be attributed to the $-\mathrm{C}=\mathrm{O}$ of PAAc. A characteristic peak around $3500 \mathrm{~cm}^{-1}$ for $\mathrm{OH}$ groups of poly acrylic acid is also appeared. This confirms that acrylic acid containing the carboxyl groups was fixed randomly onto the NWPP main chains as a grafting side chains [12-14]. Scheme 1 shows the expected structure of NWPP-g-AAc.

\subsection{Mechanical Properties}

Figures 5 and 6 show changes in percentage elongation and tensile strength at break for grafted and ungrafted NWPP films, respectively. It can be seen that the elongation percent at break for the grafted non-woven sheets decreases gradually as the grafting degree increases. However, the tensile strength increases with increasing the degree of grafting up to $66 \%$ grafting degree, thereafter, any increase in the grafting degree leads to de crease in tensile strength. The decrease in the percentage elongation

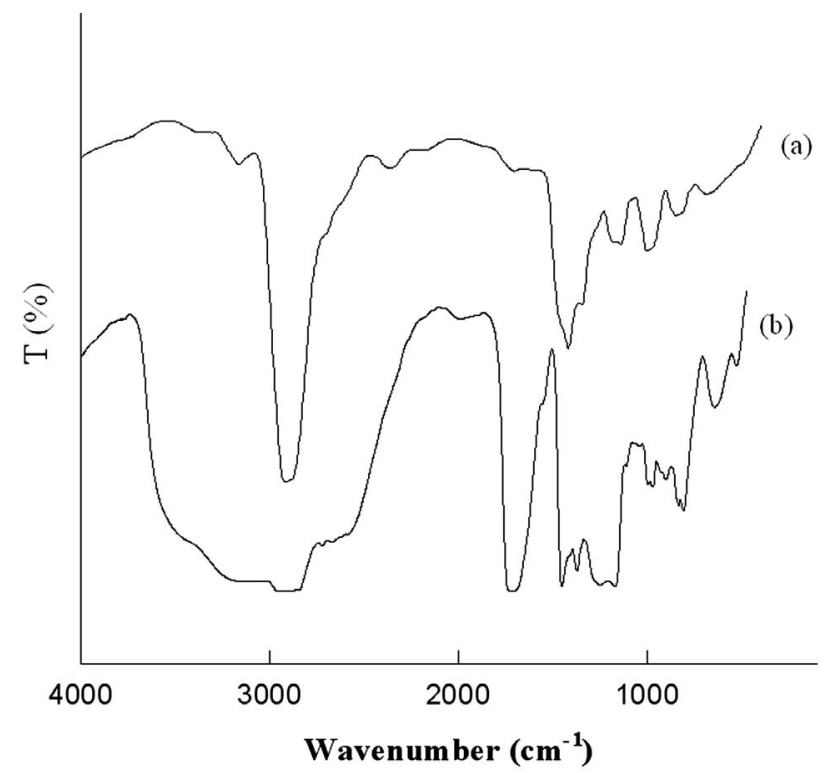

Figure 4. FTIR spectra for (a) NWPP; (b) NWPP-g-AAc. The AAc concentration ( $30 \mathrm{wt} \%$ ) and degree of grafting (64\%).

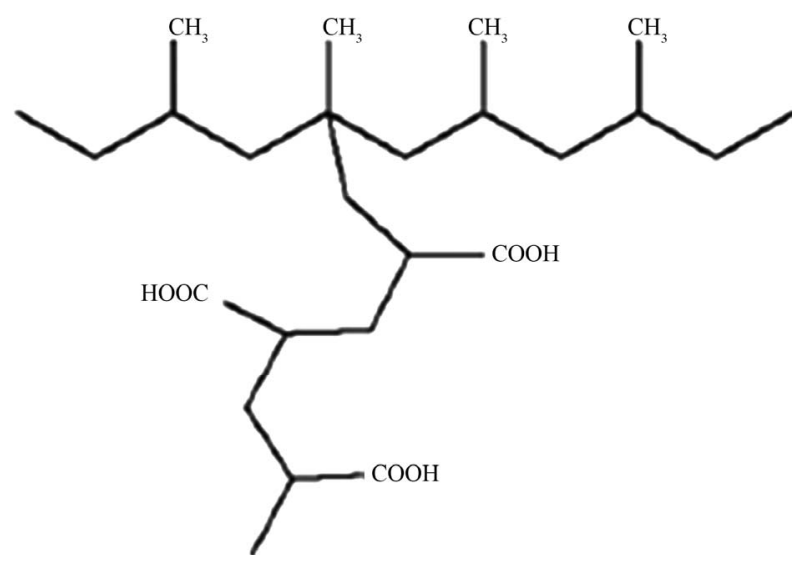

Scheme 1. The expected structure of NWPP-g-AAc

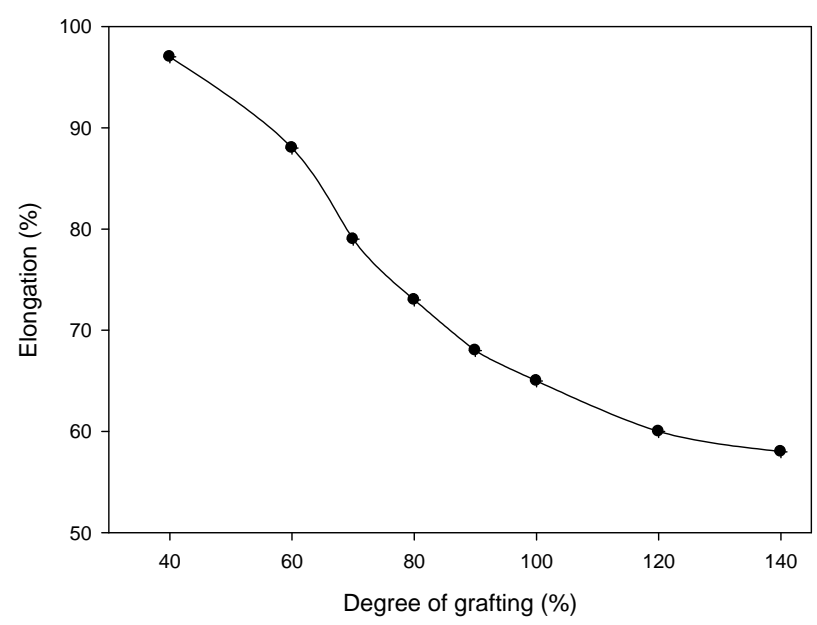

Figure 5. Elongation percent at break for NWPP-g-AAc having different degrees of grafting. 


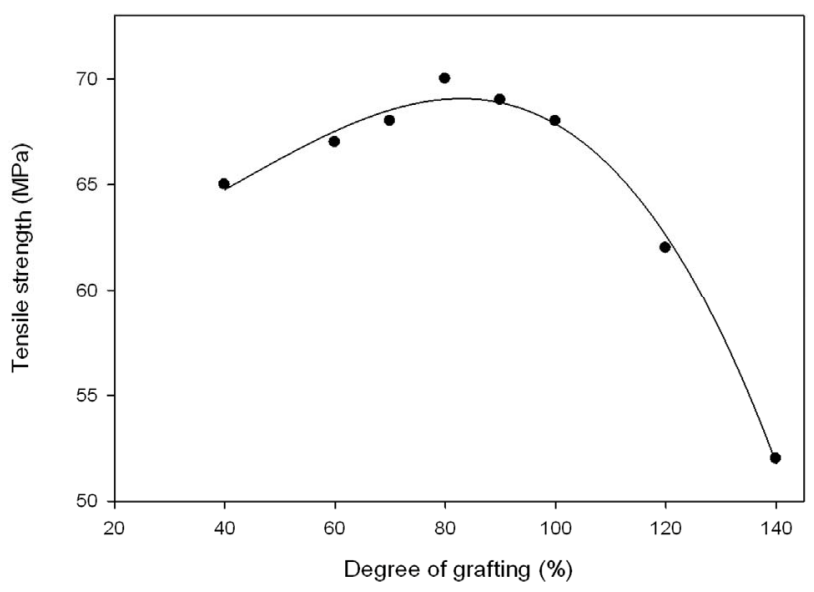

Figure 6. Tensile strength at different degrees of grafting.

and the improvement in the tensile strength is expected due to the increase of crosslinking sites with increasing the number of grafted chains and their lengths $[15,16]$. The cross-linked network structure resulted in a pronounced increase in the tensile strength of grafted sheet in the range from $60 \%-100 \%$ grafting and as consequence the percentage elongation decreased $[17,18]$. Again, the rigidity of the graft copolymer increases with an increase in degree of grafting due to two reasons; the first one is the incorporation of the polar groups and their interactions (the formation of cross-linked network structure via hydrogen bonding of carboxylic acid groups of PAAc graft chains). The second reason is the formation of crosslinking network structure, which formed because of grafting and irradiation, which leads to restriction in chain mobility [18-20]. The decrease in the tensile strength of high grafted yield non-woven sheet prepared at high irradiation dose was due to the degradation of polypropylene non-woven sheet caused at high irradiation dose.

\subsection{DSC Thermal Parameters of Grafted NWPP}

In order to determine the effect of grafting degree on the thermal parameters of the grafted NWPP, the change in Tm and $\Delta H_{m}$ was investigated by DSC technique and is shown in Table 1. The decrease in Tm and $\Delta H_{m}$ values for the grafted NWPP films compared with ungrafted ones can be attributed to the network structure, which had considerable mobility with the molten NWPP. The results obtained, suggested that the introduction of grafted chains into NWPP caused changes in its morphological structure in which the crystallinity domains in NWPP decreases due to the crosslinking network formation that caused by radiation grafting process.

\subsection{Thermogravimetric Analysis (TGA)}

The thermograms for the NWPP and grafted NWPP are
Table 1. DSC results of NWPP-g-AAc.

\begin{tabular}{ccc}
\hline Grafting degree (\%) & $T_{m}$ & $\Delta H_{m}$ \\
\hline 0 & 160 & 61.9 \\
66 & 157 & 14.5 \\
93 & 155.8 & 16.6 \\
130 & 153.8 & 5.7 \\
\hline
\end{tabular}

shown in Figure 7 and Table 2. The results show that the thermal stability of NWPP films has been changed by grafting with AAc. The grafted films exhibited two decomposition peaks, i.e., the first decomposition occurring at around $200^{\circ} \mathrm{C}-235^{\circ} \mathrm{C}$ may be due to cyclization and dehydration reactions of - $\mathrm{COOH}$ functional groups of the grafted chains. The decomposition of the second peak should be attributed to the decomposition of the grafted film and due to extensive graft chains degradation of the polymer backbone chain.

It is evident from the thermograms that the ungrafted NWPP is thermally stable up to $236^{\circ} \mathrm{C}$. The grafting of AAc onto NWPP decreases the weight loss of the major degradation step compared to that of the ungrafted NWPP as shown in Table 2. A considerable decrease in the weight loss of the major degradation step is observed systematically by increasing degree of grafting. The analysis of the thermograms shows that the degradation of grafted polymer is controlled mainly by the degree of grafting. In conclusion the introduction of AAc into NWPP sheets does not affect significantly the thermal stability of the NWPP sheets.

\subsection{X-ray Diffraction Analysis (XRD)}

X-ray diffraction of the grafted NWPP was performed and compared with the base polymer to clarify their crystallinity morphological structures changes caused by the grafting as shown in Figure 8. It is observed that the intensity of the diffractogram, representing crystallinity, decreases by grafting. This is due to dilution of the crystal line fraction by the incorporation of the amorphous PAAc chains into the polymer matrix. It can be concluded that the graft polymerization took place predominantly on the polymer film surface of the crystallites progressed inward thus breaking up partly ordered chain structures of the NWPP matrix [21].

\subsection{Selectivity of NWPP-G-Aac towards Different Metals in Mixture}

Because of functional groups, which have been introduced into polymer to be used as chelating polymer, the capacity of the polymer is an important factor to determine how much such graft copolymer is required to quantitatively 


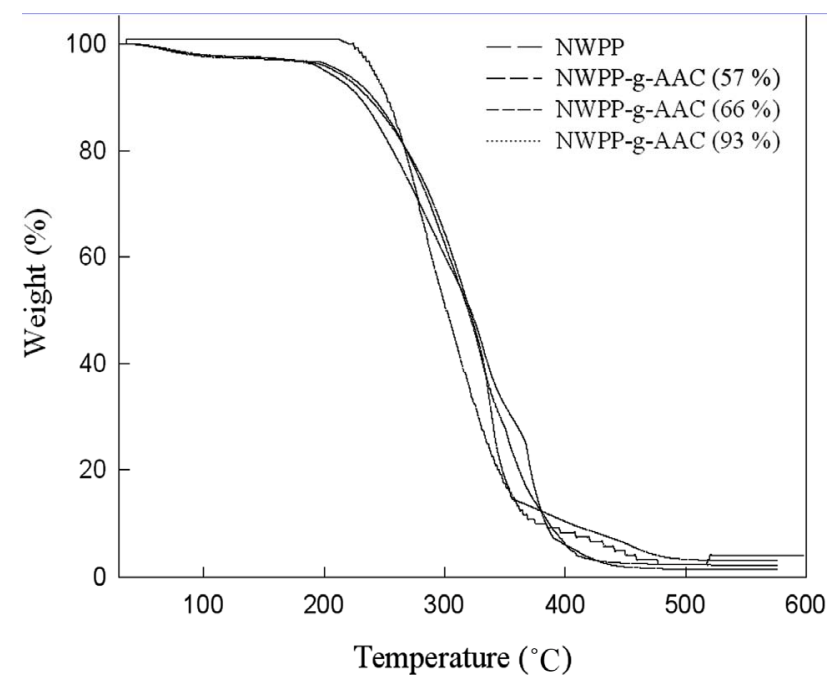

Figure 7. Thermogravimetric curves of pure NWPP and the grafted NWPP sheets.

Table 2. TGA data for PP-g-AAc.

\begin{tabular}{cccc}
\hline $\begin{array}{c}\text { Grafting } \\
\text { degree }(\%)\end{array}$ & $\begin{array}{c}\text { Onset } \\
\text { temperature }\left({ }^{\circ} \mathrm{C}\right)\end{array}$ & $\begin{array}{c}\text { Char residue } \\
(\%)\end{array}$ & $\begin{array}{c}\text { Wt. loss in the major } \\
\text { degradation step (\%) }\end{array}$ \\
\hline 0 & 236 & 2.5 & 90.5 \\
57 & 225 & 2.3 & 89.8 \\
66 & 220 & 2.1 & 87 \\
93 & 215 & 1.5 & 87 \\
\hline
\end{tabular}

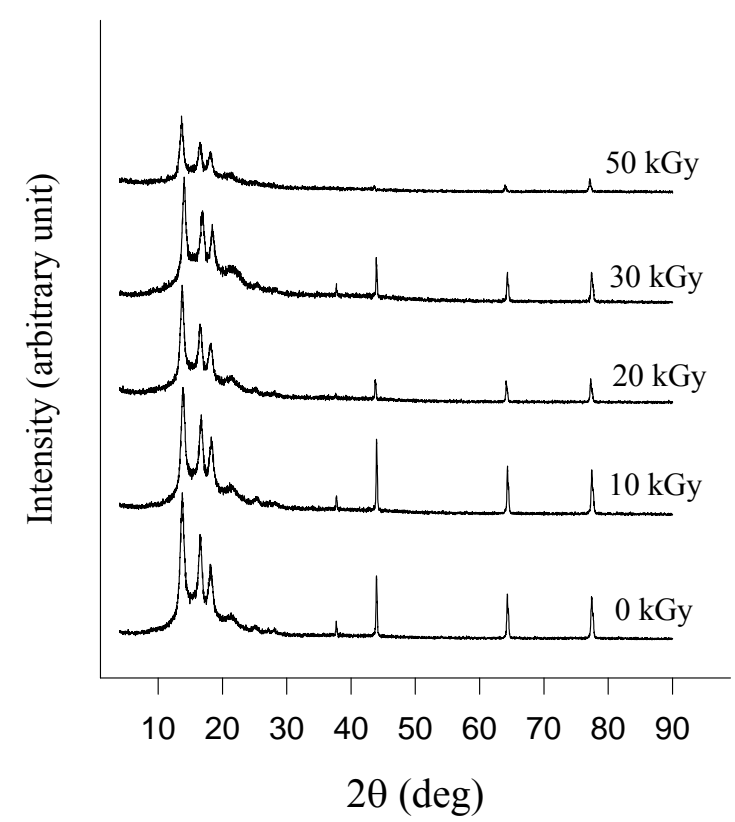

Figure 8. XRD patterns for NWPP-AAc graft copolymers prepared by gamma irradiation at different doses.

remove a specific metal ion from the solution. Thus, the capacity of NWPP-g-AAc towards different metals such as $\mathrm{Cu}, \mathrm{Co}$ and $\mathrm{Ni}$ was determined and is shown in Figure 9. The NWPP-g-AAc has a good affinity towards such metals and follows the order $\mathrm{Cu}>\mathrm{Co}>\mathrm{Ni}$ as shown in Figure 9(a). The results show that the NWPP-g-AAc has a great ability to adsorb $\mathrm{Cu}$ rather than other metals under investigation.

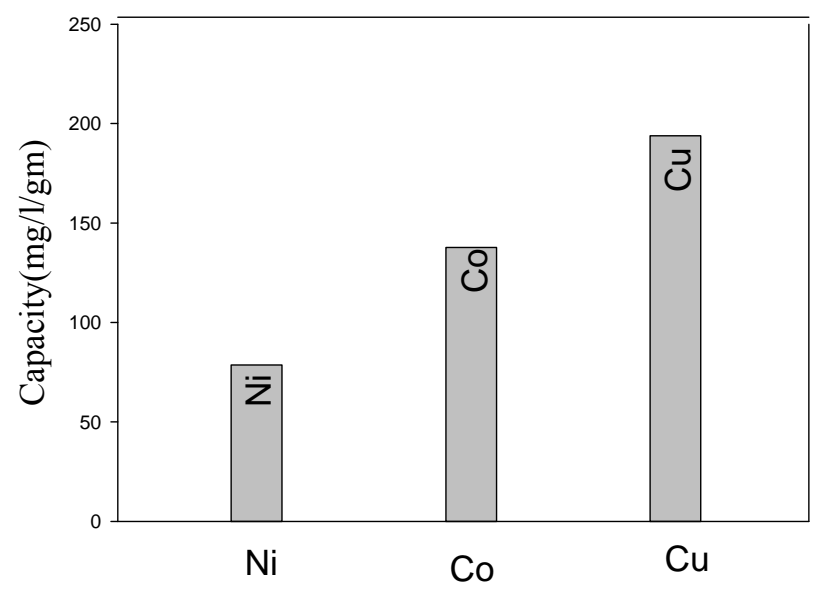

(a)

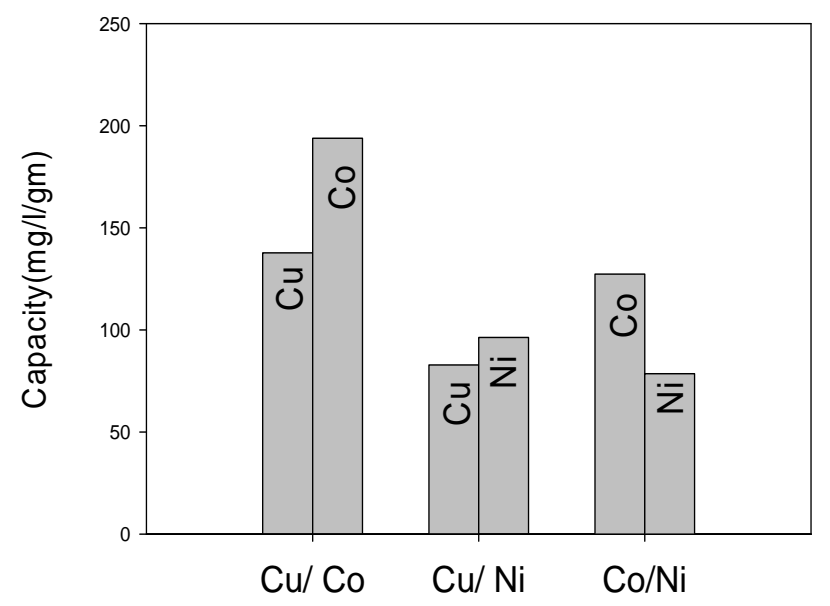

(b)

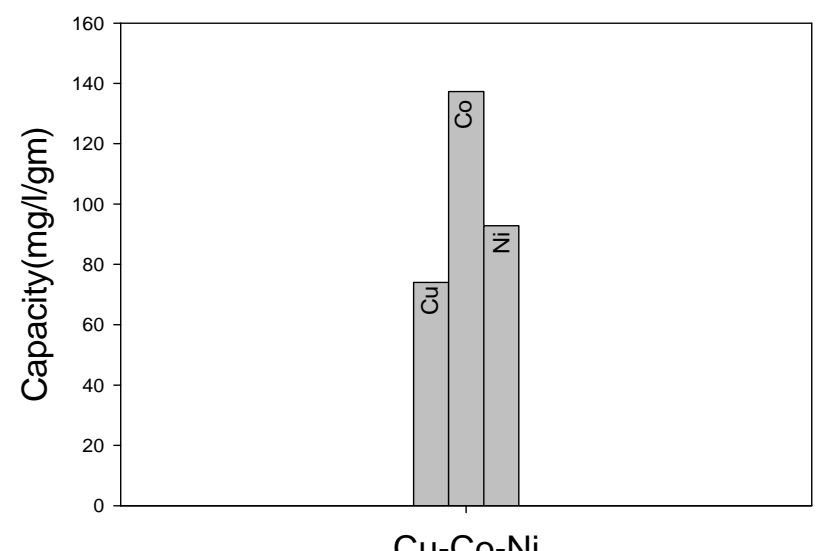

(c)

Figure 9. (a) The capacity of NWPP-g-AAc towards different metals; (b) The selectivity of $\mathrm{Cu}$ in presence of $\mathrm{Ni}$ and Co ions; (c) The selectivity of the prepared NWPP-g-AAc in a mixture Co, $\mathrm{Cu}$ and $\mathrm{Ni}$. 
The selectivity of $\mathrm{Cu}$ in presence of $\mathrm{Ni}$ and $\mathrm{Co}$ ions in a mixture is investigated using NWPP-g-AAc and the results are shown in Figure 9(b). The selectivity of the prepared NWPP-g-AAc towards $\mathrm{Cu}$ is lower than other metals ( $\mathrm{Ni}$ and $\mathrm{Co}$ ). The results show that the $-\mathrm{COOH}$ groups of AAc have a great ability to adsorb Co rather than other metals under investigation including $\mathrm{Cu}$ which has medium affinity to be adsorbed by the grafted polymer. The affinity of the prepared NWPP-g-AAc towards $\mathrm{Ni}$ is lower than Co. However, the selectivity of the prepared polymer towards $\mathrm{Cu}$ in the presence of $\mathrm{Ni}$ is lower. Also, the selectivity of the prepared polymer towards Co in the presence of $\mathrm{Cu}$ is higher.

The affinity of the prepared NWPP-g-AAc towards $\mathrm{Cu}$, $\mathrm{Ni}$ and $\mathrm{Co}$ in a mixture was investigated. It is clear that the NWPP-g-AAc prefer $\mathrm{Co}$ rather than $\mathrm{Ni}$ and $\mathrm{Cu}$. The selectivity follows the order $\mathrm{Co}>\mathrm{Ni}>\mathrm{Cu}$. This can be shown clearly from the metal uptake by NWPP/AAc copolymer Figure 9(c).

The selectivity of the prepared graft copolymer is highly dependent on the complex stability formed between the metal ion and polymer functional groups. The higher the stability of complex the higher the affinity is obtained. Among other important factors determining the stability and affinity are the metal ion radii, its electronic distribution, hydration energy, valency and ionic strength $[22,23]$.

\section{Conclusion}

The preparation of grafted nonwoven polypropylene sheets with acrylic acid monomer using ionizing radiations such as gamma rays from Co-60 was investigated. The optimum conditions for this radiation grafting system to obtain suitable grafting yield with homogeneous distribution were determined. The prepared NWPP-g-AAc is of a great interest for its applicability in industrial waste treatment from heavy and toxic metals. From the practical point of view, the applicability of the prepared NWPP-g-AAc is very promising due to its high capacity and selectivity towards different heavy metal ions such as $\mathrm{Cu}, \mathrm{Ni}$ and $\mathrm{Co}$.

\section{REFERENCES}

[1] H. Seki and A. Suzuki, "Adsorption of Heavy Metal Ions onto Insolubilized Humic Acid," Journal of Colloid and Interface Science, Vol. 171, No. 2, 1995, pp. 490-494. doi:10.1006/jcis.1995.1207

[2] A. Bhattacharya and B. N. Misra, "Grafting: A Versatile Means to Modify Polymers: Techniques, Factors and Applications," Progress in Polymer Science, Vol. 29, No. 8, 2004, pp. 767-814. doi:10.1016/j.progpolymsci.2004.05.002

[3] M. M. Nasef and E. A. Hegazy, "Preparation and Appli- cations of Ion Exchange Membranes by Radiation-Induced Graft Copolymerization of Polar Monomers onto Non-Polar Films," Progress in Polymer Science, Vol. 29, No. 6, 2004, pp. 499-561.

doi:10.1016/j.progpolymsci.2004.01.003

[4] G. MaKay, "Use of Adsorbents for the Removal of Pollutants from Wastewaters," CRC Press, Boca Raton, 1996.

[5] Y.-X. Wang, "Chinese Nonwovens Filter Media Market," Filtration \& Separation, Vol. 37, No. 10, 2000, pp. 24-25. doi:10.1016/S0015-1882(01)80093-8

[6] G. S. Bhat, "Nonwovens as Three-Dimensional Textiles for Composites," Material Manufacturing Process, Vol. 10, No. 4, 1995, pp. 667-688. doi:10.1080/10426919508935059

[7] S. H. Choi and Y. C. Nho, "Introduction of Carboxylic Acid Group to Polypropylene Fabric for Battery Separator," Korean Journal of Chemical Engineering, Vol. 16, No. 4, 1999, pp. 505-510. doi:10.1007/BF02698276

[8] H. J. Park and C. K. Na, "Preparation of Anion Exchanger by Amination of Acrylic Acid Grafted Polypropylene Nonwoven Fiber and Its Ion-Exchange Property," Journal of Colloid and Interface Science, Vol. 301, No. 1, 2006, pp. 46-54. doi:10.1016/j.jcis.2006.05.003

[9] K. S. Chen, J. C. Tsai, C. W. Chou, M. R. Yang and J. M. Yang, "Effects of Additives on the Photo-Induced Grafting Polymerization of N-Isopropylacrylamide Gel onto PET Film and PP Nonwoven Fabric Surface," Materials Science and Engineering: $C$, Vol. 20, No. 1-2, 2002, pp. 203-208. doi:10.1016/S0928-4931(02)00034-6

[10] A. Bozzi and A. Chapiro, "The Nature of the Initiating Centres for Grafting in Air-Irradiated Perfluoro Polymers," European Polymer Journal, Vol. 23, No. 3, 1987, pp. 255-257. doi:10.1016/0014-3057(87)90085-1

[11] A. Chapiro, "Radiation Chemistry of Polymeric Systems," Interscience, New York, 1962.

[12] K. Sato, S. Ikeda, M. Lida, A. Oshima, T. Tabata and M. Washio, "Study on Poly-Electrolyte Membrane of Crosslinked PTFE by Radiation-Grafting," Nuclear Instruments and Methods in Physics Research Section B: Beam Interactions with Materials and Atoms, Vol. 208, 2003, pp. 424-428. doi:10.1016/S0168-583X(03)00898-X

[13] M. M. Khalil, N. M. EL-Sawy and A. EL-Shobaky, "Gamma-Irradiation Effects on the Thermal and Structural Characteristics of Modified Grafted Polypropylene," Journal of Applied Polymer Science, Vol. 102, No. 1, 2006, pp. 506-515. doi:10.1002/app.24236

[14] B. Cheng, X. Jiao and W. Kang, "Studies on Grafting of Acrylic Acid onto Polypropylene Melt-Blown Nonwovens Induced by Electron-Beam Preirradiation," Journal of Applied Polymer Science, Vol. 102, 2006, pp. 4971-4977. doi:10.1002/app.24841

[15] H. M. Said, H. H. Sokker and A. El-Hag Ali, "Acrylation of Pre-Irradiated Polypropylene and Its Application for Removal of Organic Pollutants," Radiation Physics and Chemistry, Vol. 79, No. 4, 2010, pp. 534-539. doi:10.1016/j.radphyschem.2009.09.005

[16] H. Chen, G. R. Palmese and Y. A. Elabd, "Electrosensi- 
tive Permeability of Membranes with Oriented Polyelectrolyte Nanodomains," Macromolecules, Vol. 40, No. 4, 2007, pp. 781-782. doi:10.1021/ma062678q

[17] Y. Tang, A. Kusoglu, A. M. Karlsson, M. H. Santare, S. Cleghorn and W. B. Johnson, "Mechanical Properties of a Reinforced Composite Polymer Electrolyte Membrane and Its Simulated Performance in PEM Foil Cells," Journal of Power Sources, Vol. 175, No. 2, 2008, pp. 817-825. doi:10.1016/j.jpowsour.2007.09.093

[18] E. A. Hegazy, H. Kamal, N. A. Khalifa and Gh. A. Mahmoud, "Ion Exchange Membranes for Metal Ions Separation," Iranian Polymer Journal, Vol. 8, No. 4, 1999, pp. 223-230.

[19] M. F. Abou Taleb, Gh. A. Mahmoud, S. M. Elsigeny and E. A. Hegazy, "Adsorption and Desorption of Phosphate and Nitrate Ions Using Quaternary (Polypropylene-g-N, N-Dimethylamino Ethylmethacrylate) Graft Copolymer," Journal of Hazardous Materials, Vol. 159, No. 2-3, 2008, pp. 372-379. doi:10.1016/j.jhazmat.2008.02.028

[20] E. A. Hegazy, S. M. Mokhtar, M. B. S. Osman and A. B. Mostafa, "Study on Non-Ionic Membrane Prepared by Radiation-Induced Graft Polymerization," International
Journal of Radiation Applications and Instrumentation. Part C. Radiation Physics and Chemistry, Vol. 36, No. 3, 1990, pp. 365-370. doi:10.1016/1359-0197(90)90020-I

[21] S. Hietala, S. Holmberg, M. Karjalainen, J. Nasman, M. Paronen, R. Serimaa, F. Sundholm and S. Vahvaselka, "Structural Investigation of Radiation Grafted and Sulfonated Poly(Vinylidene Fluoride), PVDF, Membranes," Journal of Materials Chemistry, Vol. 7, No. 5, 1997, pp. 721-726. doi:10.1039/a607675k

[22] I. E. A. Hegazy, H. A. Abd El-Rehim and H. A. Shawky, "Investigation and Characterization of Radiation Grafted Copolymers for Possible Practical Use in Waste Water Treatment," Radiation Physics and Chemistry, Vol. 57, No. 1, 2000, pp. 85-95. doi:10.1016/S0969-806X(99)00312-6

[23] H. A. Abd El-Rehim, E. A. Hegazy and A. El-Hag Ali "Selective Removal of Some Heavy Metal Ions from Aqueous Solution Using Treated Polyethylene-g-Styrene/ Maleic Anhydride Membranes," Reactive \& Functional Polymers, Vol. 43, No. 1-2, 2000, pp. 105-116. doi:10.1016/S1381-5148(99)00009-7 